\title{
Nonlinear coherent optical image processing using logarithmic transmittance of bacteriorhodopsin films
}

\author{
John D. Downie
}

\begin{abstract}
The transmission properties of some bacteriorhodopsin-film spatial light modulators are uniquely suited to allow nonlinear optical image-processing operations to be applied to images with multiplicative noise characteristics. A logarithmic amplitude-transmission characteristic of the film permits the conversion of multiplicative noise to additive noise, which may then be linearly filtered out in the Fourier plane of the transformed image. I present experimental results demonstrating the principle and the capability for several different image and noise situations, including deterministic noise and speckle. The bacteriorhodopsin film studied here displays the logarithmic transmission response for write intensities spanning a dynamic range greater than 2 orders of magnitude.
\end{abstract}

\section{Introduction}

Images with multiplicative noise characteristics present special challenges for many applications including optical correlation ${ }^{1}$ and image restoration. ${ }^{2} \mathrm{~A}$ well-used approach in such situations is to transform the original noisy image logarithmically. This nonlinear operation on the image separates the noise from the underlying object function by producing a new image with additive noise. Traditional linear filtering techniques can then be applied for purposes of image restoration, reconstruction, etc. For some optical processing applications such as correlation it may be more efficient to perform the logarithmic preprocessing of the images optically. This requires an optical material that exhibits a logarithmic transfer function of some type. Bacteriorhodopsin $(B R)$ is an organically derived material that demonstrates many desirable intrinsic properties for use as a real-time optically addressed spatial light modulator for optical processing applications. ${ }^{3,4}$ These include photochromism, second-order optical nonlinearity, photoelectric effect, and photoinduced anisotropy. Some previously studied applications for BR films include Fourier-plane spatial filtering, ${ }^{5}$ optical limiting, ${ }^{6}$ time-averaged holographic interferometry, ${ }^{7}$ real-

The author is with NASA, Ames Research Center, Mail Stop 269-3, Moffett Field, California 94035.

Received 17 October 1994; revised manuscript received 15 March 1995.

0003-6935/95/235210-08\$06.00/0.

1995 Optical Society of America. time holography for optical correlation, ${ }^{8}$ and holographic imaging through a time-varying thin phase aberration. ${ }^{9}$

In a recent paper $I$ showed that certain $B R$ films exhibit a logarithmic transmission property in intensity and demonstrated the efficient conversion of a speckle image into a transformed image with the appropriate additive noise characteristics. ${ }^{10}$ In this paper I present data illustrating that the amplitudetransmission properties of some films also exhibit behavior that is logarithmic over a large dynamic range. I then use this effect to transform images in amplitude to allow subsequent coherent optical filtering or image-processing techniques to eliminate the unwanted noise that cannot be entirely removed with the same filters on the original multiplicative images. For the application of logarithmic image processing discussed here I exploit the photochromic behavior of $\mathrm{BR}$. The remainder of this paper is divided into four sections. I first describe BR and its basic physical nature. I then present the theory, procedure, and optical setup of the experiments, followed by the results from three experiments conducted to demonstrate efficient optical image processing of images with multiplicative noise. In the last section I summarize the results and present some conclusions.

\section{Bacteriorhodopsin Films}

$\mathrm{BR}$ is a protein molecule found in the photosynthetic system of a salt-marsh bacterium called Halobacterium salinarium. The BR molecule is located in a cell membrane commonly called the purple membrane. 
To the bacterium, BR is important in an oxygendeficient environment, as the BR molecules function as light-driven proton pumps that transport protons across the cell membrane. This creates a proton gradient, which in turn generates an electrochemical potential used by the organism to synthesize adenosine triphosphate. Effectively, BR is used by the bacterium to convert sunlight directly into chemical energy. The absorption of light also initiates a photocycle in the BR molecule in addition to the transportation of protons. The characteristics and effects of this photocycle make it a potentially useful material as an optically addressed spatial light modulator. This is especially true because the photocycle continues to operate after the $B R$ is extracted from the bacterium in the form of two-dimensional crystalline sheets of PM and suspended in various solutions in the preparation of films.

A schematic diagram of the BR photocycle is shown in Fig. 1. In the dark the molecule is initially in the bR state, which has an absorption spectrum peaked at $\sim 570 \mathrm{~nm}$. Upon absorption of a photon in this band the molecule quickly passes through several intermediate states via thermal relaxation until it reaches the $M$ state, in which its absorption spectrum is shifted $\sim 160 \mathrm{~nm}$ toward the blue from the initial bR state. In this state the peak absorption wavelength lies at $\sim 412 \mathrm{~nm}$, and even though the spectra of both the $b R$ and $M$ states are fairly broad, there is little overlap between them. Thus images may be written in a film of BR based on the conversion of molecules from the $b R$ state to the $M$ state. In its native form, at nominal $\mathrm{pH}$, at relative humidity, and at ambient temperature, the lifetime of the $M$ state is $\sim 10 \mathrm{~ms}$. However, the image lifetime can be increased by several orders of magnitude with chemical ${ }^{4}$ and mutagenic ${ }^{11}$ methods. The $\mathrm{M}$ state can also be stabilized by temperature control at approximately $-40{ }^{\circ} \mathrm{C}{ }^{4}$ Additionally, one can actively erase images by exposing the material in the $\mathrm{M}$ state to blue light, which directly returns the molecule to the bR state. Thus BR films can effectively be used as real-time, erasable

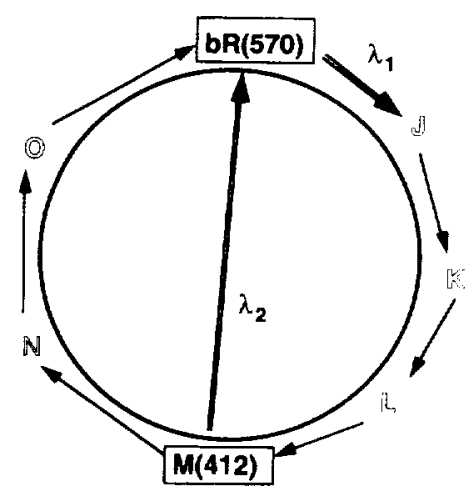

Fig. 1. Schematic of BR photocycle. The numbers in parentheses indicate peak wavelengths of the absorption spectra at the $b R$ state and the $M$ state. The transition time from $b R \rightarrow M$ is $\sim 50 \mu \mathrm{s}$, and the thermal relaxation time from $\mathrm{M} \rightarrow \mathrm{bR}$ is $\sim 10 \mathrm{~ms}$ for $B R$ in its native form. optical photographic or holographic films that require no development process, although in general the images recorded are not permanent. Other characteristics of importance for the optical image-processing application considered here include a potentially fast image-writing rate limited to $\sim 50 \mu \mathrm{s}$ by the intrinsic photocycle time and very high spatial resolution of several thousands of line pairs per millimeter due to the small molecular size of BR.

The film used in this experiment was prepared from the wild-type form of BR by Bend Research, Inc. After extraction from the bacteria, the purplemembrane material was suspended in a gelatinpolymer matrix and deposited on an optically flat substrate. The film was dried in a controlled humidity environment to lower the water content to a value of $\sim 20 \%$. A sodium hydroxide additive was employed in the matrix to raise the solution $\mathrm{pH}$ to $\sim 9.0$, which worked to lengthen the M-state lifetime (image lifetime) to $\sim 18 \mathrm{~s}$. This lifetime value is measured as the $1 / e$ point on a curve of transmitted intensity at $633 \mathrm{~nm}$ versus time after initial bleaching. Practically speaking, the effective lifetime is even longer because the lifetime decay curve shows a multiexponential decay behavior rather than a simple singleexponential decay. This means that more BR molecules remained in the $M$ state after the 1 / $e$ point then is predicted by a single-exponential decay, thus lengthening the effective lifetime. The film was sealed between the substrate and another glass plate to protect the film and ensure a uniform thickness across the 25-mm-diameter aperture. Index-matching fluid was placed between the film and glass surfaces to ensure high optical quality and prevent unwanted reflections. The film thickness was $\sim 100 \mu \mathrm{m}$ and the optical density of the film in the bR state at the absorption peak wavelength was 2.5 .

The light-transmission characteristics of the film at one wavelength were measured experimentally with a variable-strength write beam and a weak read beam whose intensity was small enough that it would not contribute to the bleaching effect, or photoconversion, and thus not affect the transmission measurements. The experimental setup is shown schematically in Fig. 2. Both write and readout beams are plane waves from an argon-ion $\left(\mathrm{Ar}^{+}\right)$laser at $514.5 \mathrm{~nm}$. The film is first exposed to the write beam, which causes part of the bR-state population to traverse the photocycle and switch to the $M$ state. In a steadystate condition the read beam is passed through the

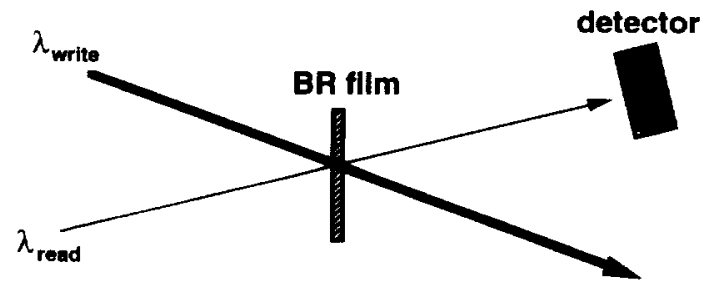

Fig. 2. Schematic of the optical setup for making measurements of transmission characteristics of BR film. The wavelengths of the write beam and the readout beam were both $514.5 \mathrm{~nm}$. 
exposed area of the film, and the transmitted intensity is measured with a photodetector. The data describing the relationship between transmitted amplitude or modulus of the read beam $A_{\text {trans,read }}$ and the incident power density of the write beam $E_{\text {write }}$ are given in Fig. 3. The transmitted-amplitude data were generated by taking the square root of the measured transmitted-intensity data. It is clear that the amplitude-transmission function is very close to logarithmic over most of the range of write-beam power density; i.e.,

$$
A_{\text {trans,read }}=K \log \left(E_{\text {write }}\right)+c
$$

for some constants $K$ and $c$. Thus in general the intensity and amplitude transmittances of the weak readout beam are controlled by the power density of the write beam. This is of course the principle on which the basis of using BR films as optically addressed spatial light modulators is founded. For various applications the write and read beams may be either coherent or incoherent. For example, one can obtain incoherent-to-coherent light conversion by making the write beam incoherent and reading out with a weak coherent beam. For our application here of preprocessing images with multiplicative noise for subsequent optical image processing the read beam is again coherent, and the write beam may be either. For the more specific case of treating speckle images, both the writing and reading beams are coherent. The transmittance relation displayed in Fig. 3 and expressed in Eq. (1) shows that this film exhibits a logarithmic amplitude transfer function, which is exactly that required for the proper transformation of images with multiplicative noise such that the image component and the noise component are additive in the Fourier-transform plane. Additionally, the dynamic range of the write power over which this property holds is large ( $>2$ orders of magnitude). However, the transmittance relation depends on many variables including film thickness, optical density, M-state lifetime, and readout wavelength. ${ }^{5,12}$ Films with different characteristics will display significantly different transmission properties.

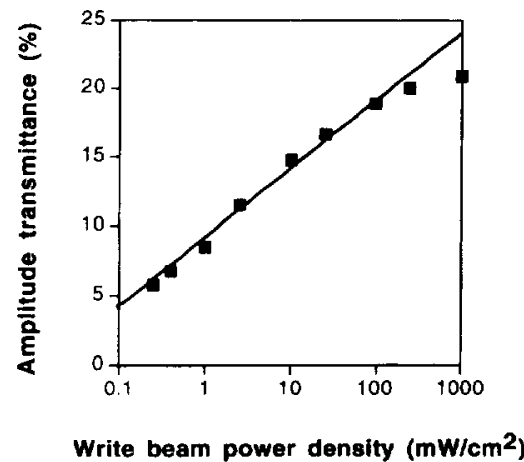

Fig. 3. Transmittance of read-beam amplitude as a function of write-beam power density for wild-type bacteriorhodopsin film. Both beams have wavelengths of $514.5 \mathrm{~nm}$. The square boxes are experimentally measured data points, and the solid black line is the best-fit logarithmic curve.

\section{Image-Processing Theory and Optical Setup}

When a signal $s(x, y)$ is corrupted by additive noise $n(x, y)$ such that the observed quantity $r(x, y)=$ $s(x, y)+n(x, y)$, it is at least theoretically possible optically to filter out the noise in the Fourier plane of a coherent optical processor, provided that the Fourier transforms $S(u, v)$ and $N(u, v)$ are sufficiently separated in the frequency domain. ${ }^{13}$ However, if the observed image $r(x, y)$ is multiplicative in nature, such as

$$
r(x, y)=s(x, y) n(x, y),
$$

then filtering techniques in the Fourier plane of a coherent optical processor become much less effective because the image transform is given by

$$
R(u, v)=S(u, v) \otimes N(u, v),
$$

where $\otimes$ represents the convolution operator. In this case the Fourier transform $S(u, v)$ and $N(u, v)$ are convolved and impossible to separate, making it much more difficult to isolate the original or intended signal $s(x, y)$. In general, there are many examples of images that contain multiplicative noise, including speckle images formed with coherent radiation illuminating a rough object, and uneven conventional illumination of an arbitrary object. I will consider both examples here in the experiments.

A well-known approach to the processing of images with multiplicative noise is the application of a logarithmic transformation that separates the signal and noise to make them additive. ${ }^{14,15}$ In this case we have

$$
\log [r(x, y)]=\log [s(x, y)]+\log [n(x, y)]
$$

and then traditional Fourier-domain filtering can be applied to the transform of $\log [r(x, y)]$ to remove as much noise as possible. The resultant image $\log [s(x, y)]$ can either be viewed or used as it is or possibly transformed again in an exponential manner to recover $s(x, y)$, if desired. While the logarithmictransformation and filtering operations are relatively straightforward to accomplish digitally, there may be circumstances in which it is desirable to perform the filtering operation optically, such as with the input of images to an optical correlator system for patternrecognition purposes. If we term the filtering operation as the processing of the image, then the logarithmic transformation to make the signal and noise additive is essentially a preprocessing step. For an all-optical processing system we would of course want to perform the logarithmic operation optically as well. The transmission properties of the BR film studied here allow the easy implementation of this transformation in a parallel fashion with a simple and versatile optical system.

The logarithmic preprocessing transformation has been previously implemented optically with a halftonescreen process. ${ }^{16}$ While this technique produced logarithmic amplitude transmission over a dynamic range 
of 2.0 in optical density and the subsequent imageprocessing experiments yielded good experimental results, this approach does not offer the prospect of real-time operation. There is necessarily a long time delay involved owing to the exposure, removal, and development of the photographic film placed in contact with the logarithmic halftone screen. The halftone negative is then photoreduced to form a halftone positive, which is replaced back in the optical system. While useful for a single image, this procedure would not be practical for the processing of many different images rapidly. Another technique was also recently presented for the specific recovery of a signal from speckle noise. ${ }^{17}$ This approach uses two photorefractive-crystal phase conjugators in series to produce a quadratic nonlinear processor. While this system produces the signal with an additive noise term and does not suffer from the development delays of the halftone-screen approach, it also does not yield a purely logarithmic transformation. This is clear because the additive noise produced from the original speckle pattern is still signal dependent in nature, while a logarithmic transform would convert the speckle noise to additive signal-independent noise. ${ }^{18,19}$ Moreover, the double phase-conjugator system is significantly more complex than the simple bacteriorhodopsin system that we present here.

The optical system used in our image-processing experiments is shown in Fig. 4. An argon-ion $\left(\mathrm{Ar}^{+}\right)$ laser beam at wavelength $514.5 \mathrm{~nm}$ is collimated and then split into two arms with a nonpolarizing beam splitter (BS), thus preserving the same vertical linear polarization in each beam. Each beam is individually controlled with an electronic shutter. With shutter A open and shutter B closed the write beam is passed through the object, which consists of an image pattern with multiplicative noise. Lens $L_{1}$ images the object function onto the BR film. The power level of the laser is adjusted to ensure that the write-beam power density at the BR-film plane is within the central logarithmic region of the transmission curve shown in Fig. 3 . The image is encoded in real time through the photoconversion of $\mathrm{BR}$ molecules through the photocycle from the bR ground state to the $M$ state. Shutter $A$ is then closed, shutter B is opened, and we pass the much-weaker

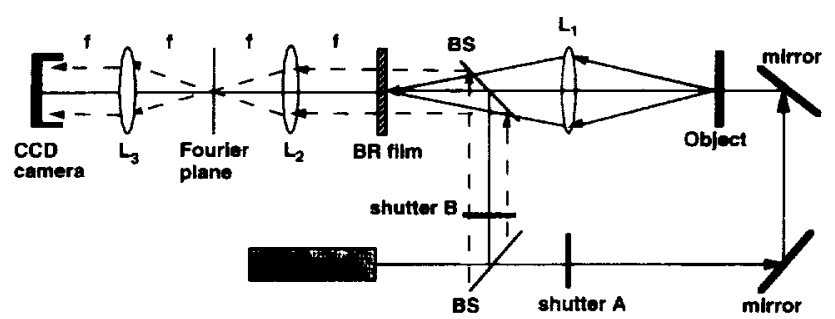

Fig. 4. Optical setup for logarithmic transformation and subsequent Fourier-plane spatial filtering of images with multiplicative noise. Shutter $A$ is opened during the image-recording process in BR film, and shutter B is opened during the read process and the image-processing operation. The argonion ( $\mathrm{Ar}^{-}$) laser wavelength used is $514.5 \mathrm{~nm}$. read beam through the $B R$ film. Lens $L_{2}$ performs the Fourier transform of the transmitted field through the BR film, and lens $L_{3}$ (having the same focal length $f$ as $\mathrm{L}_{2}$ ) reimages the transmitted read beam into the CCD camera. In general, a linear filter is inserted at the Fourier plane to filter out the unwanted noise that is the Fourier transform of $\log [n(x, y)]$, as given in Eq. (4).

It is important to note at this point that the overall transmission properties of the BR film are strongly dependent on the wavelength of the read beam. Not only may the modulus or amplitude $(A=\mid$ intensity $\mid)$ transmittance as shown in Fig. 3 change significantly for different wavelengths, but the transmitted phase of the read beam is also wavelength dependent in general. This is important because the photochromic property of BR films generally produce index-ofrefraction changes in addition to the absorption spectrum shift. This means that, not only will the read beam experience different local amplitude transmittance for varying write-beam intensity, but in general the local refractive index will also depend somewhat on the write-beam intensity. For holography applications the refractive-index modulation is usually desirable because the total diffraction efficiency is the sum of the individual amplitude and phase grating components. However, for the present application of logarithmic image transformation, we would prefer to avoid any significant variation of the phase transmittance. We want to effect only the logarithmic amplitude-transmission behavior because concurrent refractive-index variations may alter the expected image Fourier-transform distribution. This could potentially adversely affect our ability to adequately filter out the unwanted noise term. We can easily show that the effect in the Fouriertransform plane of the image read out through the BR film is the convolution of the Fourier transform of the encoded phase pattern with the Fourier transforms of the additive logarithms of the signal and noise terms.

In this regard the optimal wavelength to use for reading out the image encoded in the $B R$ film would be $568 \mathrm{~nm}$, provided of course that the amplitude transmittance at this wavelength remains logarithmic with respect to write intensity. This is because previous studies have shown that the expected indexof-refraction difference $\Delta n$ between the bR and M states at $568 \mathrm{~nm}$ is zero. ${ }^{20,21}$ Not having access to this wavelength in my laboratory, I therefore assessed the effect of the phase variations using a $514.5-\mathrm{nm}$ read beam. A film of very similar composition (wild-type BR material, 2.5 optical density) was previously analyzed theoretically and in simulation in Ref. 20. From these data I estimated the refractiveindex variations $\Delta n$ experienced at $514.5 \mathrm{~nm}$ to be $\leq 0.0005$, which for a 100 - $\mu$ m-thick film corresponds to transmitted phase variations $\Delta \phi<\pi / 5$. These variations are small, and I performed some simple but representative computer simulations that verified that they are generally not a factor in the image- 
processing application studied here. The experimental results presented in Section 4 also provide direct evidence that readout at $514.5 \mathrm{~nm}$ is feasible and valid with this film.

\section{Experimental Results}

The first image-processing experiment conducted to demonstrate the logarithmic processing capability of the BR film involves an original image composed of two crossed Ronchi gratings. The dynamic range of the image (for transmission to light at $514.5 \mathrm{~nm}$ ) is $\sim 1.5$ in density. The original image and the image transmitted by the BR film are shown in Fig. 5. The original image appears nearly binary because it is the multiplication of two binary grating patterns. On the other hand the logarithmic image passed by the BR film clearly transmits some light between the brightest areas, which is more representative of an addition of the two gratings. The Fourier spectra of these two images are presented in Fig. 6. The spectrum of the original image contains cross-term components obtained from the convolution of the two individual grating spectra, and these components do not appear in the spectrum of the transformed image. This is again indicative of an additive image pattern.

I next performed a simple image-processing experiment on both images. Using the system in Fig. 4, I placed a simple spatial filter in the Fourier-transform plane to block the components of the vertical grating (vertical lines) in an effort to remove it from the image. The filter is shown schematically in Fig. 7. It blocks only the components from the purely vertical grating. The results from this filtering operation are shown in Fig. 8. The image obtained after filtering of the original multiplicative image is pre-

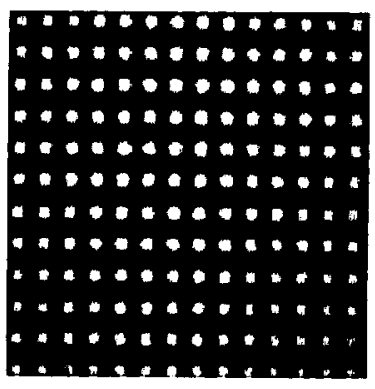

(a)

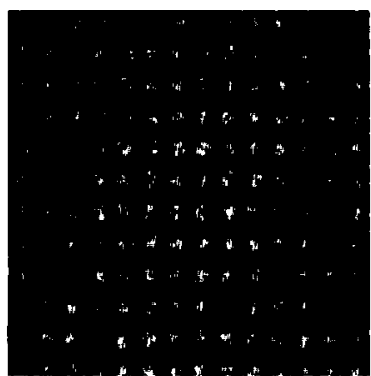

(b)

Fig. 5. (a) Original unfiltered input pattern of multiplicative crossed gratings; (b) unfiltered image of the logarithmically transformed crossed-gratings pattern transmitted by BR film.

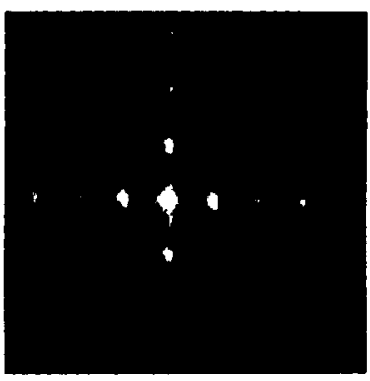

(a)

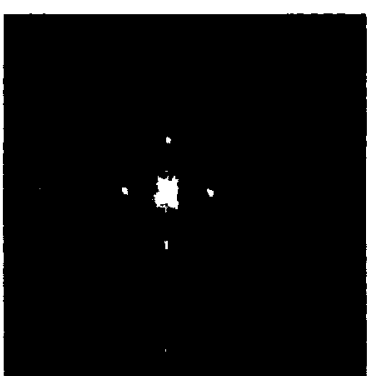

(b)

Fig. 6. (a) Fourier spectrum of the original multiplicative crossedgrating image; (b) Fourier spectrum of the logarithmically transformed crossed-grating image.

sented in Fig. 8(a). The vertical grating is clearly not completely removed from this image because the filter allows transmittance of the convolutional cross terms that still contains information of that image component. However, as seen in Fig. 8(b), we are able to remove the vertical grating completely from the logarithmic image using this filter. This is because no cross terms are present in the Fourier transform, and thus the vertical grating is completely blocked by the filter while passing the horizontal grating component spectrum.

The second experiment performed is similar in that the original image is a binary image of an $\mathrm{X}$ multiplied by a horizontal grating pattern. The original and logarithmically transformed images are shown in Fig. 9. The original is clearly binary in nature, and the image transmitted by the BR film is again representative of an image composed of an addition of the logarithms of the $\mathrm{X}$ and the grating. In the optical system I turned the image on its side such that the $\mathrm{X}$ was horizontal and the grating was vertical. This

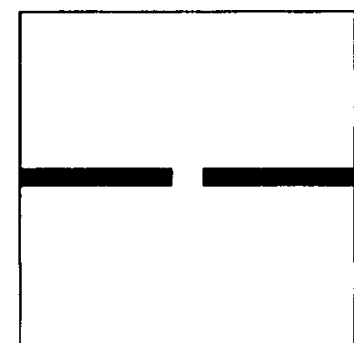

Fig. 7. Fourier-plane filter used in image-processing experiments of the original and the logarithmically transformed versions of the crossed-gratings pattern. 


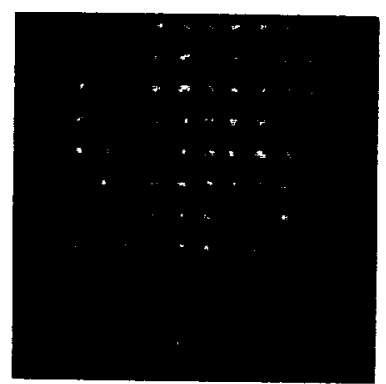

(a)

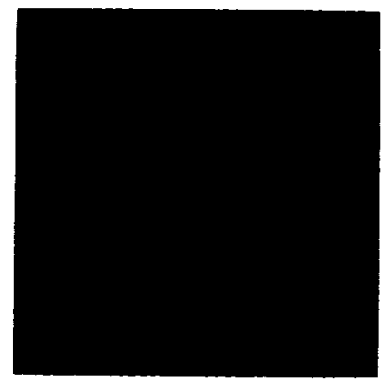

(b)

Fig. 8. (a) Output image after a filtering operation in the Fourier plane of the original multiplicative crossed-grating image; (b) output image after a filtering operation in the Fourier plane of the logarithmically transformed version of the crossed-grating image

allowed use of the same spatial filter of Fig. 7 to process the two images to remove the grating as much as possible. The resulting images as shown in Fig. 10 were rotated $90^{\circ}$ after capture, as were the images in Fig. 9. Because the spectrum of the original image is the convolution of the spectra of the $\mathrm{X}$ and the grating, I was not able to remove the grating completely from the image with this filter. How-

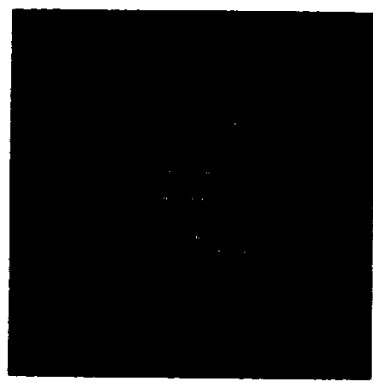

(a)

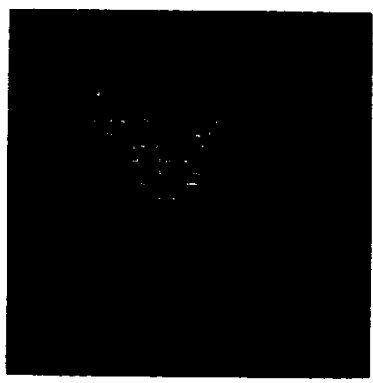

(b)

Fig. 9. (a) Original unfiltered input pattern of an X multiplied by a horizontal grating; (b) unfiltered image of the logarithmically transformed version of the same pattern transmitted by BR film.

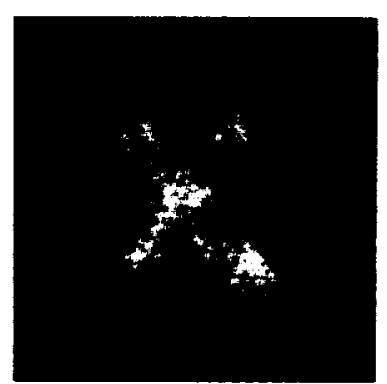

(a)

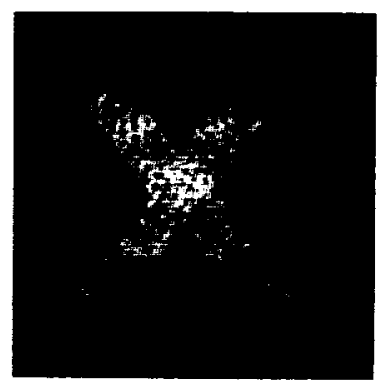

(b)

Fig. 10. (a) Output image after a filtering operation in the Fourier plane of the original pattern of $\mathrm{X}$ multiplied by a horizontal grating; (b) output image after a filtering operation in the Fourier plane of the logarithmically transformed version of the same pattern.

ever, in Fig. 10(b) we see that the grating is removed from the image transmitted by the BR film, demonstrating that the logarithmic transformation performed by the BR film makes the two image components additive rather than multiplicative.

The final demonstration experiment involves the filtering of an image corrupted by speckle noise. Speckle is a commonly occurring type of multiplicative image noise that is found during imaging with spatially coherent light or radiation when the object of interest has a random surface roughness on the order of a wavelength and the imaging system cannot resolve the microscale of the object's roughness. ${ }^{22}$ Speckle can be observed in images from coherent synthetic aperture radar, laser and sonar imaging systems, ultrasonic imaging systems that employ coherent acoustic irradiation, and holographic and interferometric images, to name only a few examples. The statistical properties of speckle noise have been thoroughly studied in the past, and it is well known that the image intensity generally follows a negative exponential probability-density function. ${ }^{23}$ An image model that has been widely used to describe speckle images is the multiplicative model

$$
r_{\mathrm{sp}}(x, y)=s(x, y) n_{\mathrm{sp}}(x, y)
$$

where $r_{\mathrm{sp}}(x, y)$ is the noisy speckle image intensity, $s(x, y)$ is the incoherent intensity image of the object, and $n_{\mathrm{sp}}(x, y)$ is the noise function. ${ }^{15,16}$ Equation (5) is strictly true only for spatially uniform areas of the object and is a less accurate model for image regions that contain spatial details smaller than the resolution of the coherent system. ${ }^{24}$ Assuming the model 
of Eq. (5), and negative exponential statistics for $r_{\mathrm{sp}}(x, y)$, then the random variable $n_{\mathrm{sp}}(x, y)$ necessarily also has a negative exponential probability-density function:

$$
p_{\mathrm{n}}\left[n_{\mathrm{sp}}(x, y)\right]=\exp \left[-n_{\mathrm{sp}}(x, y)\right] .
$$

It is then straightforward to show that both the mean and the standard deviation of $n_{\mathrm{sp}}(x, y)$ are equal to 1 , implying that the mean and the standard deviation of $r_{\mathrm{sp}}(x, y)$ are both equal to $s(x, y)$. A logarithmic transformation of an image described by Eq. (5) will change the noise from multiplicative to additive, as

$$
\log \left[r_{\mathrm{sp}}(x, y)\right]=\log [s(x, y)]+\log \left[n_{\mathrm{sp}}(x, y)\right]
$$

and presumably the additive noise suffered by this transformed image can be filtered out to a greater degree than the multiplicative noise in the original speckle image. This transformation not only serves to make the noise additive but the noise standard deviation also becomes constant and no longer related to the mean. ${ }^{18,19}$ I have previously verified this behavior of the transmittance of a BR film. ${ }^{10}$ Of course, to obtain the exact transformation of Eq. (7) for an arbitrary original speckle pattern, we would require that the logarithmic transformation of the BR film possess an infinitely large dynamic range. This is obviously impossible, but fortunately Kato and Goodman found that, for an original pattern (before submersion in speckle noise) with a dynamic range of 1 order of magnitude or less, then a logarithmic transformation with a dynamic range of 2.0 orders of magnitude will be sufficient to change the speckle noise to additive noise. ${ }^{16}$ Figure 3 shows that the dynamic range of the BR-film logarithmic transformation is greater than $\mathbf{2 . 0}$ orders of magnitude and should thus be adequate for this purpose.

The speckle image that I used in the experiment with filtering speckle noise is shown in Fig. 11(a). I obtained this by placing the binary $X$ pattern in contact with a transparent random-phase object. The image of the logarithmically transformed pattern transmitted by the BR film after recording is shown in Fig. 11(b). The noise in this image is no longer speckle noise but instead has the characteristics of random additive zero-mean noise. The height-towidth ratio of these images are different from thase in Figs. 9 and 10 because, as noted previously, I obtained the images in the earlier figures by placing the $\mathrm{X}$ on its side and then rotating the captured images $90^{\circ}$. The rectangular geometry of the CCD camera pixels accounts for the difference in aspect ratio.

I then filtered both the original and transformed images. The filter used in both cases was a simple low-pass circular aperture placed at the Fourier plane whose diameter was adjusted to be just large enough to pass the Fourier transform of the $\mathrm{X}$ object and to block all higher spatial frequencies. The resulting images obtained after the filtering operations are shown in Fig. 12. We are unable to filter out the speckle noise from the original image because of the

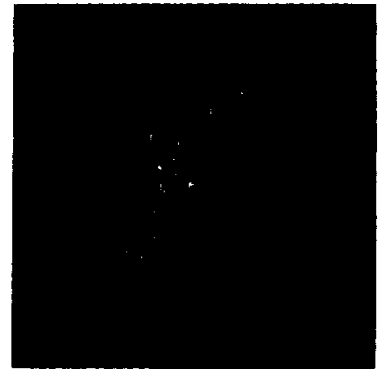

(a)

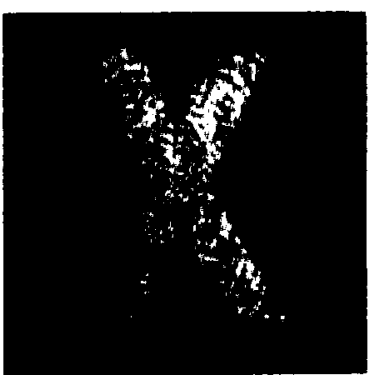

(b)

Fig. 11. (a) Original unfiltered input pattern of an $\mathrm{X}$ with speckle noise; (b) unfiltered image of the logarithmically transformed version of the same pattern transmitted by BR film.

convolution of the noise spectrum with the spectrum of the $\mathrm{X}$ object. Instead, this filtering operation merely seems to increase the scale size of the speckle noise in the processed image of Fig. 12(a). On the other hand we are able to filter out a substantial amount of the noise from the logarithmically transformed image, as shown in Fig 12(b). There is a type of interference fringe noise superimposed on this

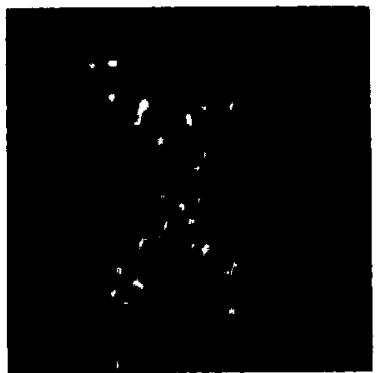

(a)

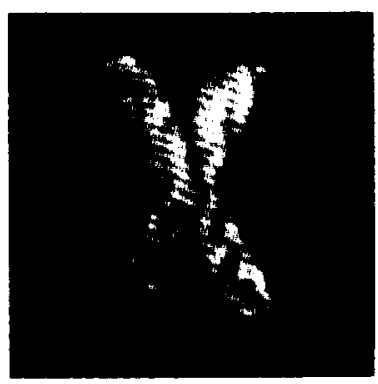

(b)

Fig. 12. (a) Output image after a filtering operation in the Fourier plane of the original pattern of $X$ with speckle noise; (b) output image after a filtering operation in the Fourier plane of the logarithmicălly transformed version of the same pattern. 
image that is an artifact of the optical system. Ignoring this artifact, we can see that the processed logarithmic image in Fig. 12(b) is relatively uniform in intensity, demonstrating the ability to filter out much of the additive noise with the simple filter. This again confirms the satisfactory performance of the BR film acting as an amplitude logarithmic transformer.

\section{Summary and Conclusions}

I have demonstrated the use of a bacteriorhodopsin film as an optical logarithmic image transformer with application to coherent optical image processing of images with multiplicative noise properties. The photochromic behavior of the BR film was shown to possess logarithmic amplitude-transmission characteristics over a dynamic range of greater than 2.0 orders of magnitude. In the experiments, images with multiplicative noise were first preprocessed optically with the BR film to produce transformed images with additive noise characteristics. Both these transformed images and the original images were subjected to identical Fourier-plane coherent spatialfiltering operations. Although the filtering was largely unsuccessful with the original images owing to the convolutional relationship of the signal and noise spectra, the logarithmically transformed images were indeed successfully processed to eliminate much of the noise. Given the real-time recording and erasure capabilities of $\mathrm{BR}$, this material may be well suited for high-throughput optical image-processing operations for images with multiplicative noise such as speckle. This would be especially important for all-optical systems such as optical correlators in which it might be advantageous and efficient to perform the image processing optically as well as the subsequent pattern-recognition operation.

This work was supported by the NASA Office of Advanced Concepts and Technology under RTOP 233-02-05-06. The BR films were made by Bend Research, Inc., Bend, Oregon.

\section{References}

1. J. D. Downie and J. F. Walkup, "Optimal correlation filters for images with signal-dependent noise," J. Opt. Soc. Am. A 11, 1599-1609 (1994)

2. J. F. Walkup and R. C. Choens, "Image processing in signal dependent noise," Opt. Eng. 13, 258-266 (1974).

3. C. Bräuchle, N. Hampp, and D. Oesterhelt, "Optical applications of bacteriorhodopsin and its mutated variants," Adv. Mater. 3, 420-428 (1991).

4. R. R. Birge, "Photophysics and molecular electronic applications of the rhodopsins," Ann. Rev. Phys. Chem 41, 683-733 (1990).

5. R. Thoma, N. Hampp, C. Bräuchle, and D. Oesterhelt, "Bacte- riorhodopsin films as spatial light modulators for nonlinearoptical filtering," Opt. Lett. 16,651-653 (1991).

6. Q.W. Song, C. Zhang, R. Gross, and R. Birge, "Optical limiting by chemically enhanced bacteriorhodopsin films," Opt. Lett. 18, 775-777 (1993).

7. T. Renner and N. Hampp, "Bacteriorhodopsin-films for $d y$ namic time average interferometry," Opt. Commun. 96, 142149 (1993).

8. N. Hampp, R. Thoma, D. Oesterhelt, and C. Bräuchle, "Biological photochrome bacteriorhodopsin and its genetic variant ASp96 $\rightarrow$ Asn as media for optical pattern recognition," Appl. Opt. 31, 1834-1841(1992).

9. J. D. Downie, "Real-time holographic image correction using bacteriorhodopsin," Appl. Opt. 33, 4353-4357 (1994).

10. J. D. Downie, "Optical logarithmic transformation of speckle images with bacteriorhodopsin films," Opt. Lett. 20, 201-203 (1995).

11. A. Miller and D. Oesterhelt, "Kinetic optimization of bacteriorhodopsin by aspartic acid 96 as an internal proton donor," Biochim. Biophys. Acta 1020, 57-64 (1990).

12. Q. W. Song, C. Zhang, R. Blumer, R. B. Gross, Z. Chen, and R. R. Birge, "Chemically enhanced bacteriorhodopsin thinfilm spatial light modulator," Opt. Lett. 18, 1373-1375 (1993).

13. J. W. Goodman, Introduction to Fourier Optics (McGraw-Hill, New York, 1968), Chap. 7, p. 142.

14. A. V. Oppenheim, R. W. Schafer, and T. G. Stockham, Jr., "Nonlinear filtering of multiplied and convolved signals," Proc. IEEE 56, 1264-1291 (1968).

15. H. H. Arsenault and G. April, "Properties of speckle integrated with a finite aperture and logarithmically transformed," J. Opt. Soc. Am. 66, 1160-1163 (1976).

16. H. Kato and J. W. Goodman, "Nonlinear filtering in coherent optical systems through halftone screen processes," Appl. Opt. 14, 1813-1824 (1975).

17. J. Khoury, A. M. Biernacki, C. L. Woods, and M. CroninGolomb, "Photorefractive quadratic processor for signal recovery from multiplicative complex noise," Opt. Eng. 32, 2872 2876 (1993).

18. H. H. Arsenault and M. Denis, "Integral expression for transforming signal-dependent noise into signal-independent noise," Opt. Lett. 6, 210-212 (1981).

19. P. R. Prucnal and B. E. A. Saleh, "Transformation of imagesignal-dependent noise into image-signal-independent noise,' Opt. Lett. 6, 316-318 (1981).

20. R. B. Gross, K. C. Izgi, and R. R. Birge, "Holographic thin films, spatial light modulators and optical associative memories based on bacteriorhodopsin," in Image Storage and Retrieval Systems, A. A. Jamberdino and W. Niblack, eds., Proc. Soc. Photo-Opt. Instrum. Eng. 1662, 186-196 (1992).

21. D. Zeisel and N. Hampp, "Spectral relationship of lightinduced refractive index and absorption changes in bacteriorhodopsin films containing wild-type $\mathrm{BR}_{\mathrm{WT}}$ and the variant BR $_{\text {D96N }}, " J$ J. Phys. Chem. 96, 7788-7792 (1992).

22. J. W. Goodman, Laser Speckle and Related Phenomena, J. C. Dainty, ed., Vol. 9 of Topics in Applied Physics ISpringerVerlag, New York, 1975), p. 10.

23. J. W. Goodman, Statistical Optics (Wiley, New York, 1985), Chap. 7, p. 350.

24. M. Tur, K. C. Chin, and J. W. Goodman, "When is speckle noise multiplicative?" Appl. Opt. 21, 1157-1159 (1982). 